Article

\title{
Multilevel Coding for the Full-Duplex Decode-Compress-Forward Relay Channel
}

\author{
Ahmed Attia Abotabl and Aria Nosratinia * \\ Department of Electrical \& Computer Engineering, The University of Texas at Dallas, \\ Richardson, TX 75080, USA; ahmed.abotabl@utdallas.edu \\ * Correspondence: aria@utdallas.edu
}

Received: 14 August 2017; Accepted: 30 October 2017; Published: 14 November 2017

\begin{abstract}
The Decode-Compress-Forward (DCF) is a generalization of Decode-Forward (DF) and Compress-Forward (CF). This paper investigates conditions under which DCF offers gains over DF and $\mathrm{CF}$, addresses the problem of coded modulation for DCF, and evaluates the performance of DCF coded modulation implemented via low-density parity-check (LDPC) codes and polar codes. We begin by revisiting the achievable rate of DCF in discrete memoryless channels under backward decoding. We then study coded modulation for the decode-compress-forward via multi-level coding. We show that the proposed multilevel coding approaches the known achievable rates of DCF. The proposed multilevel coding is implemented (and its performance verified) via a combination of standard DVB-S2 LDPC codes, and polar codes whose design follows the method of Blasco-Serrano.
\end{abstract}

Keywords: decode-and-forward; compress-and-forward; multilevel coding

\section{Introduction}

Shortly after the introduction of the three-node relay channel by Van Der Meulen [1], Cover and El-Gamal [2] proposed and analyzed block-Markov encoded decode-forward (DF) and compress-forward (CF) for the relay channel [3-5]. Decode-forward is capacity achieving for the degraded relay channel, but, due to the relay decoding constraint, it does not perform well when the source-relay link is weak. Compress-forward can also be optimal under certain conditions [6], but it also falls short under certain other conditions [3,4]. This motivated a generalization of DF and CF into a hybrid technique by Cover and El-Gamal ([2], Theorem 7), denoted decode-compress-forward (DCF). This technique, its performance and implementation via coded modulation are the subjects of this paper. Other hybrid relaying protocols include hybrid Decode-forward and Amplify-forward [7], and also a variation of DCF has appeared in the context of selective cooperation [8].

The known achievable rate of DCF in the additive white Gaussian noise (AWGN) full-duplex relay channel reduces to either $\mathrm{DF}$ or $\mathrm{CF}$ achievable rates. This is a result due to [9] that we re-derive in the following under backward decoding. In the discrete input full-duplex relay channel, we show via examples that the DCF performance can exceed both DF and CF.

A coding implementation for the DCF in the AWGN channel is then proposed based on multilevel coding (MLC). Multilevel coding was proposed by Imai and Hirakawa in [10]. More details about the performance and the design of MLC can be found in [11-13]. Duan et al. [14] showed that MLC with linear mapping does not require active shaping to achieve the capacity. MLC was extended to the MIMO (multiple-input multiple-output) transmission [15], and was used for diversity coding [16-19] and in data storage [20]. Much less is known about MLC in the context of multi-node networks. Exceptions exist, for example, Hern and Narayanan [21] used MLC in the context of compute and forward and Chen et al. [22] studied multilevel coding in the two-way relay channel.

At the source and the relay, the proposed DCF multilevel coding decomposes the overall coded modulation into a group of binary codes, each either operating via a DF or a CF protocol. The mapper 
combines these constituent level-wise codes into a hybrid DF-CF coded modulation. The assignment of each level to either DF or CF and the rate allocation to each level is an optimization problem. We demonstrate the operation of this system by an implementation that employs for the DF components the DVB-S2 LDPC codes [23], and, for the CF components, a group of polar codes that are designed according to Blasco-Serrano [24,25].

\section{Preliminaries}

\subsection{The Relay Channel}

The capacity of the full-duplex relay channel is in general unknown. The capacity of the degraded relay channel is achieved by decode-forward, and achievable rates for several other protocols have also been calculated including partial decode-forward and compress-forward. In a full-duplex relay, these protocols are implemented via block-Markov encoding [26], a consequence of the causality constraint on the relay.

Throughout the paper, we denote the signal transmitted from the source node and the relay node in block $t$ by $X_{1}^{(t)}$ and $X_{2}^{(t)}$. We begin with the received signal model at the relay, which experiences self-interference:

$$
Y_{2}^{(t)}=H_{12} X_{1}^{(t)}+n_{2}+n_{s}
$$

where $H_{12}$ is the channel coefficient from the source to the relay, $n_{2}$ is the additive Gaussian (thermal) noise at the receiver, and $n_{s}$ is the sampled residual self-interference. The area of modeling and analyzing loop-back or self-interference has experienced rapid growth in the past few years. Several methods for mitigating self-interference are now in place, among them antenna design and placement (including passive components), as well as echo cancellation in the amplifier stage, as well as digital signal processing after down-conversion and sampling [27]. The collection of these methods has allowed the residual self-interference to be reduced significantly. The residue of self-interference, $n_{s}$, is the component that is seen by the relay decoder. Several works to date [27-29] have used a Gaussian model for $n_{s}$, an approximation that is confirmed by various measurements [30,31]. Therefore, the combination $\tilde{n}_{2}=n_{2}+n_{S}$ is also Gaussian with the appropriate variance.

Thus, the received signal at the relay and destination in block $t$ are respectively given by

$$
\begin{aligned}
& Y_{2}^{(t)}=H_{12} X_{1}^{(t)}+\tilde{n}_{2,} \\
& Y_{3}^{(t)}=H_{13} X_{1}^{(t)}+H_{23} X_{2}^{(t)}+n_{3},
\end{aligned}
$$

where $H_{12}, H_{13}$ and $H_{23}$ are the channel coefficients as illustrated in Figure 1. The channel coefficients can include a path-loss component as well as any type of fading.

The destination uses either backward decoding or a sliding window decoding. The former requires waiting until a terminating point where no new message is transmitted from the source, and, in the latter, the decoder uses a window of $L$ blocks for decoding.

In decode-forward, the relay decodes the message from the source in block $t$ and assists the source in its transmission during block $t+1$. In compress-forward, the relay does not attempt decoding but quantizes the received signal $Y_{2}$ and sends the quantized version to the destination.

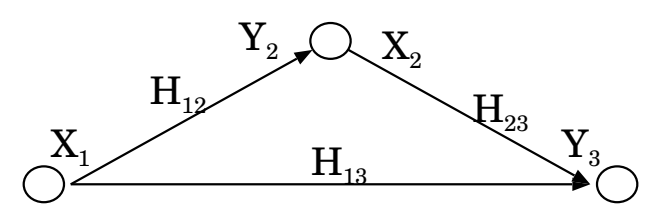

Figure 1. Full-Duplex relay channel. 


\subsection{Multilevel Coding}

In the point-to-point channel, multilevel coding (see Figure 2) divides the data stream into $m=\log _{2}(q)$ binary sub-streams for a $q$-ary constellation. Each sub-stream $i$ is encoded independently with rate $R_{i}$. At each time instance, the outputs of the (binary) encoders are combined to construct the vector $\left[B_{1}, B_{2}, \ldots B_{m}\right]$, which is then mapped to a constellation point $X$. The channel is described by the conditional distribution $P_{Y \mid X}(y \mid x)$, where $Y$ is the output of the channel. The mutual information between the input and output is given by

$$
I(X ; Y)=I\left(B_{1}, B_{2}, \ldots, B_{m} ; Y\right)=\sum_{i=1}^{m} I\left(B_{i} ; Y \mid B^{i-1}\right),
$$

where we use the chain rule of mutual information, one-to-one relationship between $X$ and $\left[B_{1}, B_{2}, \ldots, B_{m}\right]$, and the notation $B^{i-1} \equiv\left[B_{1}, B_{2}, \ldots, B_{i-1}\right]$. This equation suggests a multistage decoding where the codeword of level $i$ is decoded using the output of the decoders of the preceding levels. A necessary and sufficient condition for multilevel coding achieving the constellation constrained capacity is that the optimal distribution $P_{B_{1}, \ldots, B_{m}}^{*}\left(b_{1}, \ldots, b_{m}\right)$ must be independent across its components [32]:

$$
P_{B_{1}, \ldots, B_{m}}\left(b_{1}, \ldots, b_{m}\right)=\prod_{i=1}^{m} P_{B_{i}}\left(b_{i}\right) .
$$

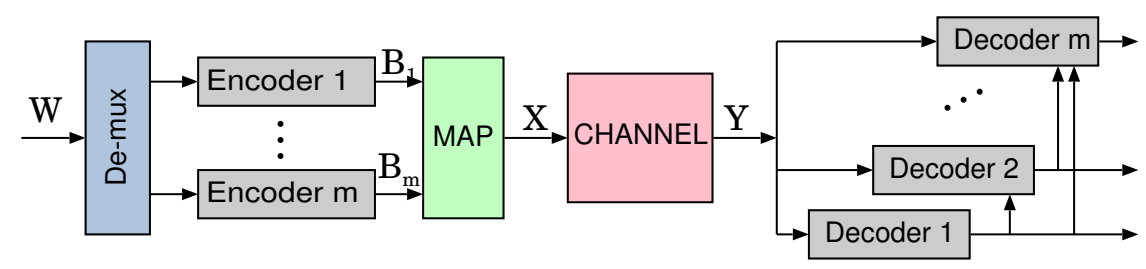

Figure 2. MLC and multi-stage decoding in the point to point channel.

\section{Decode-Compress-Forward}

In this section, we re-derive Theorem 7 [2] for the discrete memoryless relay channel under backward decoding and obtain the DCF achievable rate for the AWGN relay channel as well as the constellation constrained AWGN relay channel.

\subsection{Discrete Memoryless Full-Duplex Relay}

Block Markov encoding for decode-compress forward is shown in Figure 3 over four transmission blocks. In each transmission block, the source and the relay send a compress-forward component that is superimposed on a decode-forward component. A detailed system design and analysis is explained as follows:

In $g$ transmission blocks, a sequence of $(g-1)$ i.i.d. messages $W_{j} \in\left[1: 2^{n R}\right], j \in[1: g-1]$ is transmitted. Each message $W_{j}$ is split into two messages $W_{d j} \in\left[1: 2^{n R_{d}}\right]$ and $W_{c j} \in\left[1: 2^{n R_{c}}\right]$ for $j \in[1: g-1]$. This implies that $R=R_{d}+R_{c}$, where $R$ is the total rate, and $R_{d}, R_{c}$, respectively, indicate the rate carried by decode-forward and compress-forward components of signaling. 
BLOCK 1

SOURCE

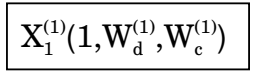

$\mathrm{X}_{1}^{(2)}\left(\mathrm{W}_{\mathrm{d}}^{(1)}, \mathrm{W}_{\mathrm{d}}^{(2)}, \mathrm{W}_{\mathrm{c}}^{(2)}\right)$

$\mathrm{X}_{1}^{(3)}\left(\mathrm{W}_{\mathrm{d}}^{(2)}, \mathrm{W}_{\mathrm{d}}^{(3)}, \mathrm{W}_{\mathrm{c}}^{(3)}\right)$

BLOCK 3

BLOCK 4

\begin{tabular}{|c|c|c|c|c|}
\hline \multirow{3}{*}{ RELAY } & $\mathrm{Y}_{2}^{(1)}$ & $\mathrm{Y}_{2}^{(2)}$ & $\mathrm{Y}_{2}^{(3)}$ & $\mathrm{Y}_{2}^{(4)}$ \\
\hline & $\hat{\mathrm{Y}}_{2}^{(1)}\left(\mathrm{Y}_{2}^{(1)}, 1,1\right)$ & $\hat{\mathrm{Y}}_{2}^{(2)}\left(\mathrm{Y}_{2}^{(2)}, \mathrm{W}_{\mathrm{d}}^{(1)}, \hat{\mathrm{Y}}_{2}^{(1)}\right)$ & $\hat{\mathrm{Y}}_{2}^{(3)}\left(\mathrm{Y}_{2}^{(3)}, \mathrm{W}_{\mathrm{d}}^{(2)}, \hat{\mathrm{Y}}_{2}^{(2)}\right)$ & $\hat{\mathrm{Y}}_{2}^{(4)}\left(\mathrm{Y}_{2}^{(4)}, \mathrm{W}_{\mathrm{d}}^{(2)}, \hat{\mathrm{Y}}_{2}^{(2)}\right)$ \\
\hline & $\mathrm{X}_{2}^{(1)}(1,1)$ & $\mathrm{X}_{2}^{(2)}\left(\mathrm{W}_{\mathrm{d}}^{(1)}, \hat{\mathrm{Y}}_{2}^{(1)}\right)$ & $\mathrm{X}_{2}^{(3)}\left(\mathrm{W}_{\mathrm{d}}^{(2)}, \hat{\mathrm{Y}}_{2}^{(2)}\right)$ & $\mathrm{X}_{2}^{(4)}\left(\mathrm{W}_{\mathrm{d}}^{(3)}, 1\right)$ \\
\hline
\end{tabular}

Figure 3. Decode-Compress-Forward transmission over four transmission blocks.

\section{Codebook generation:}

For each block $j \in[1: g]$, randomly and independently generate $2^{n R_{d}}$ sequences $U_{2 d}^{n}\left(w_{d(j-1)}\right)$ according to

$$
\prod_{i=1}^{n} P_{U_{2 d}}\left(u_{2 d i}\right)
$$

For each $w_{d(j-1)} \in\left[1: 2^{n R_{d}}\right]$, randomly and conditionally independently generate $2^{n R^{\prime}}$ sequences $X_{2}^{n}\left(l_{j-1} \mid w_{d(j-1)}\right), l_{j-1} \in\left[1: 2^{n R^{\prime}}\right]$, each according to

$$
\prod_{i=1}^{n} P_{X_{2} \mid U_{2 d}}\left(x_{2 i} \mid u_{2 d i}\left(w_{d(j-1)}\right)\right)
$$

For each $l_{j-1} \in\left[1: 2^{n R^{\prime}}\right]$, randomly and conditionally independently, generate $2^{n R^{\prime \prime}}$ sequences $\hat{Y}_{2}^{n}\left(k_{j} \mid l_{j-1}\right), k_{j} \in\left[1: 2^{n R^{\prime \prime}}\right]$ each according to

$$
\prod_{i=1}^{n} P_{\hat{Y}_{2} \mid X_{2 i}}\left(\hat{y}_{2 i} \mid x_{2 i}\left(l_{j-1} \mid w_{d(j-1)}\right)\right.
$$

$R^{\prime \prime}$ represents the compression rate at the relay. In addition, for each $w_{d(j-1)}$, randomly and conditionally independently generate $2^{n R_{d}}$ sequences $U_{1 d}^{n}\left(w_{d j} \mid w_{d(j-1)}\right), w_{d j} \in\left[1: 2^{n R_{d}}\right]$, each according to

$$
\prod_{i=1}^{n} P_{U_{1 d} \mid U_{2 d}}\left(u_{1 d i} \mid u_{2 d i}\left(w_{d(j-1)}\right)\right)
$$

Finally, for each pair of messages $w_{d(j-1)}$ and $w_{d j}$, randomly and conditionally independent generate $2^{n R_{c}}$ sequences $X_{1}^{n}\left(w_{c j} \mid w_{d(j)}, w_{d(j-1)}\right)$, each according to

$$
\prod_{i=1}^{n} P_{X_{1} \mid U_{2 d}, U_{1 d}}\left(x_{1 i} \mid u_{2 d i}\left(w_{d(j-1)}\right), u_{1 d i}\left(w_{d(j-1)}\right)\right) .
$$

This defines the codebooks

$$
\mathcal{C}_{j}=\left\{x_{1}^{n}\left(w_{c j} \mid w_{d j}, w_{d(j-1)}\right), x_{2}^{n}\left(l_{j} \mid w_{d(j-1)}\right)\right\}, \quad j \in[1: g] .
$$




\section{The source node:}

In block $j$, the pair of messages $w_{d j}$ and $w_{c j}$ are to be transmitted. The encoder at the source node chooses $x_{1}\left(w_{c j} \mid w_{d j}, w_{d(j-1)}\right)$ from codebook $\mathcal{C}_{j}$. The messages of the last block are considered to be $w_{c g}=w_{d g}=1$.

\section{The relay node:}

The decoding phase of the relay uses typicality decoding as follows: first, assume that $\tilde{w}_{d 0}=1$. Second, at the end of block $j$, the relay finds a unique $\tilde{w}_{d j}$ such that

$$
\left(x_{1}^{n}\left(w_{c j} \mid \tilde{w}_{d j}, \tilde{w}_{d(j-1)}\right), x_{2}^{n}\left(l_{j-1} \mid \tilde{w}_{d(j-1)}\right), y_{2}^{n}(j)\right) \in \mathcal{T}_{\epsilon}^{(n)}
$$

for any $w_{c j}$, where $\mathcal{T}_{\epsilon}^{(n)}$ denotes the jointly typical sets of the corresponding random variables.

The relay then finds $k_{j}$ such that

$$
\left(y_{2}^{n}(j), \hat{y}_{2}^{n}\left(k_{j} \mid l_{j-1}\right), x_{2}^{n}\left(l_{j-1}\right)\right) \in \mathcal{T}_{\epsilon}^{(n)},
$$

and if there is more than one $k_{j}$, the relay selects one at random and if the relay does not find any $k_{j}$, then, it selects one uniformly at random from $\left[1: 2^{n R^{\prime \prime}}\right]$. Based on $k_{j}$, the relay determines $l_{j}$ as it is the bin index of $k_{j}$.

In the transmission phase, in block $j$, the relay chooses $x_{2}\left(l_{j-1} \mid w_{d(j-1)}\right)$ from codebook $\mathcal{C}_{j}$.

\section{The destination node:}

The destination uses backward decoding, so it waits until the reception of the $g$ blocks and then starts decoding from the last block and successively toward the first block. For $j=g-1, g-2, \ldots, 1$, the destination finds estimates $\hat{w}_{d(j)}$ and $\hat{l}_{j}$ such that

$$
\left(x_{1}^{n}\left(\hat{w}_{c(j+1)} \mid \hat{w}_{d(j+1)}, \hat{w}_{d(j)}\right), x_{2}^{n}\left(\hat{l}_{j} \mid \hat{w}_{d(j)}\right), y_{3}^{n}(j+1)\right) \in \mathcal{T}_{\epsilon}^{(n)},
$$

where $w_{c g}=w_{d g}=1$. The destination then finds an estimate $\hat{w}_{c j}$ such that

$$
\left(x_{1}\left(\hat{w}_{c(j)} \mid \hat{w}_{d(j)}, \hat{w}_{d(j-1)}\right), x_{2}\left(\hat{l}_{j-1} \mid \hat{w}_{d(j-1)}\right), \hat{y}_{2}^{n}\left(\hat{k}_{j} \mid \hat{l}_{j-1}\right)\right) \in \mathcal{T}_{\epsilon}^{(n)}
$$

for some $\hat{k}_{j}$ that belongs to the bin $\hat{l}_{j}$.

\section{Probability of error analysis:}

Without loss of generality, the source messages are $w_{d j}=w_{c j}=1$ for $j \in[1: g]$. In block $j$, there are two error events at the relay, an error event when the relay does not decode $w_{d j}$ correctly and another when the relay makes an error in the compress-forward part. The two error events at the relay in block $j$ are defined as follows:

$$
\begin{gathered}
\tilde{\mathcal{E}}_{1}(j)=\left\{\hat{W}_{d j} \neq 1\right\}, \\
\tilde{\mathcal{E}}_{2}(j)=\left\{\left(X_{2}^{n}\left(L_{j-1} \mid W_{d(j-1)}\right), \hat{Y}_{2}^{n}\left(k_{j} \mid L_{j-1}\right), Y_{2}^{n}(j)\right) \notin \mathcal{T}_{\epsilon}^{(n)} \text { for all } k_{j} \in\left[1: 2^{n R^{\prime \prime}}\right]\right\},
\end{gathered}
$$

while the error events at the destination are defined as follows:

$$
\begin{gathered}
\mathcal{E}(j+1)=\left\{\left(W_{d(j+1)} \neq 1\right) \cup\left(W_{c(j+1)} \neq 1\right)\right\}, \\
\mathcal{E}^{\prime}(j+1)=\left\{L_{c(j+1)} \neq 1\right\}, \\
\mathcal{E}_{1}(j)=\left\{\left(X_{1}^{n}\left(\hat{W}_{c(j+1)} \mid \hat{W}_{d(j+1)}, \hat{W}_{d j}\right), X_{2}^{n}\left(\hat{L}_{j} \mid \hat{W}_{d j}\right), Y_{3}^{n}(j+1)\right) \notin \mathcal{T}_{\epsilon}^{(n)}\right\}, \\
\mathcal{E}_{2}(j)=\left\{\left(X_{1}^{n}\left(\hat{W}_{c(j+1)} \mid \hat{W}_{d(j+1)}, \hat{w}_{d j}\right), X_{2}^{n}\left(\hat{l}_{j} \mid \hat{w}_{d j}\right), Y_{3}^{n}(j+1)\right) \in \mathcal{T}_{\epsilon}^{(n)} \text { for some } \hat{w}_{d j} \neq 1, \hat{l}_{j} \neq 1\right\}, \\
\mathcal{E}_{3}(j)=\left\{\left(X_{1}^{n}\left(\hat{W}_{c(j+1)} \mid \hat{W}_{d(j+1)}, \hat{W}_{d j}\right), X_{2}^{n}\left(\hat{l}_{j} \mid \hat{W}_{d j}\right), \hat{Y}_{2}^{n}\left(\hat{K}_{j} \mid \hat{L}_{j-1}\right)\right) \notin \mathcal{T}_{\epsilon}^{(n)}\right\},
\end{gathered}
$$


$\mathcal{E}_{4}(j)=\left\{\left(X_{1}^{n}\left(w_{c(j+1)} \mid \hat{W}_{d(j+1)}, \hat{W}_{d j}\right), X_{2}^{n}\left(\hat{l}_{j} \mid \hat{W}_{d j}\right), \hat{Y}_{2}^{n}\left(\hat{K}_{j} \mid \hat{L}_{j-1}\right)\right) \in \mathcal{T}_{\epsilon}^{(n)}\right.$ for some $\left.w_{c(j+1)} \neq 1\right\}$

The probability of error is then

$$
\begin{aligned}
P(\mathcal{E}(j)) & =P\left(\tilde{\mathcal{E}}_{1}(j) \cup \tilde{\mathcal{E}}_{2}(j) \cup \mathcal{E}(j+1) \cup \mathcal{E}^{\prime}(j+1) \cup \mathcal{E}_{1}(j) \cup \mathcal{E}_{2}(j) \cup \mathcal{E}_{3}(j) \cup \mathcal{E}_{4}(j)\right) \\
& \leq P\left(\tilde{\mathcal{E}}_{1}(j)\right)+P\left(\tilde{\mathcal{E}}_{2}(j)\right)+P(\mathcal{E}(j+1)) \\
& +P\left(\left(\mathcal{E}_{1}(j) \cup \mathcal{E}_{3}(j)\right) \cap \tilde{\mathcal{E}}_{1}^{c}(j) \cap \tilde{\mathcal{E}}_{2}^{c}(j) \cap \mathcal{E}^{c}(j+1) \cap \mathcal{E}^{c}(j+1)\right)+P\left(\mathcal{E}_{2}(j)\right)+P\left(\mathcal{E}_{4}(j)\right),
\end{aligned}
$$

where the superscript ${ }^{c}$ denotes the complementary event. By the law of large numbers and the packing lemma, $P\left(\tilde{\mathcal{E}}_{1}(j)\right) \rightarrow 0$ as $n \rightarrow \infty$ if

$$
R_{d} \leq I\left(U_{1 d} ; Y_{2} \mid U_{2 d}\right)
$$

By independence of the codebooks and the covering lemma, the term $P\left(\tilde{\mathcal{E}}_{2}(j)\right) \rightarrow 0$ as $n \rightarrow \infty$ if

$$
R^{\prime \prime} \geq I\left(\hat{Y}_{2} ; Y_{2} \mid X_{2}\right)
$$

For $P(\mathcal{E}(j+1))$, since the messages of the last block is known exactly to be 1 , by induction and satisfying the other constraints, $P(\mathcal{E}(j+1)) \rightarrow 0$ as $n \rightarrow \infty$.

By the independence of the codebooks and the law of large numbers, the term $P\left(\left(\mathcal{E}_{1}(j) \cup \mathcal{E}_{3}(j)\right) \cap\right.$ $\left.\tilde{\mathcal{E}}_{1}^{c}(j) \cap \tilde{\mathcal{E}}_{2}^{c}(j) \cap \mathcal{E}^{c}(j+1) \cap \mathcal{E}^{c}(j+1)\right) \rightarrow 0$ as $n \rightarrow \infty$. The term $P\left(\mathcal{E}_{2}(j)\right) \rightarrow 0$ as $n \rightarrow \infty$ if

$$
\begin{aligned}
& R_{d} \leq I\left(U_{1 d}, U_{2 d} ; Y_{3}\right), \\
& R^{\prime} \leq I\left(X_{2} ; Y_{3} \mid U_{2 d}\right), \\
& R_{c} \leq I\left(X_{1} ; \hat{Y}_{2}, Y_{3} \mid X_{2}, U_{1 d}\right) .
\end{aligned}
$$

Eventually, the total transmission rate is $R_{d}+R_{c}$. By combining the previous rate constraints, we have:

Theorem 1. The achievable rate of decode-compress-forward is given by

$$
R \leq \min \left\{I\left(U_{1 d} ; Y_{2} \mid U_{2 d}\right), I\left(U_{1 d}, U_{2 d} ; Y_{3}\right)\right\}+I\left(X_{1} ; \hat{Y}_{2}, Y_{3} \mid X_{2}, U_{1 d}\right)
$$

subject to

$$
I\left(Y_{2} ; \hat{Y}_{2} \mid X_{2}, U_{1 d}\right) \leq I\left(X_{2} ; Y_{3}\right)-I\left(U_{2 d} ; Y_{3}\right)
$$

where

$$
\begin{aligned}
& P_{Y_{3}, Y_{2}, \hat{Y}_{2}, U_{1 d}, U_{2 d}, X_{1}, X_{2}}\left(y_{3}, y_{2}, \hat{y}_{2}, u_{1 d}, u_{2 d}, x_{1}, x_{2}\right) \\
& \quad=P_{U_{2 d}}\left(u_{2 d}\right) P_{U_{1 d} \mid U_{2 d}}\left(u_{1 d} \mid u_{2 d}\right) P_{X_{1} \mid U_{1 d}}\left(x_{1} \mid u_{1}\right) P_{X_{2} \mid u_{2 d}}\left(x_{2} \mid u_{2}\right) P_{Y_{2} \mid X_{1}}\left(y_{2} \mid x_{1}\right) \\
& \quad P_{\hat{Y}_{2} \mid U_{1 d}, X_{2}, Y_{2}}\left(\hat{y}_{2} \mid u_{1}, x_{2}, y_{2}\right) P_{Y_{3} \mid X_{1}, X_{2}}\left(y_{3} \mid x_{1}, x_{2}\right) .
\end{aligned}
$$

\subsection{AWGN Full-Duplex Relay}

Assume that all the variables in the Section 3.1 are Gaussian, and the source and relay have an average power constrained by $P_{1}$ and $P_{2}$, respectively. Note that Gaussian codebooks are not known to 
be optimal in this case. A block-Markov encoding of DCF in the AWGN channel is shown in Figure 4 under four transmission blocks. The source and relay signals are given by

$$
\begin{aligned}
& X_{1}=U_{1 d}+\beta U_{2 d}+U_{1 c} \\
& X_{2}=U_{2 d}+U_{2 c}
\end{aligned}
$$

respectively.

Block 1

Source

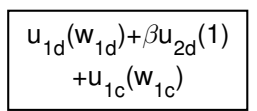

Block 3

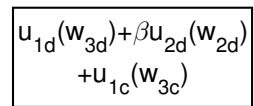

$$
u_{2 d}\left(w_{2 d}\right)+u_{2 c}\left(w_{2 c}\right)
$$

Block 4

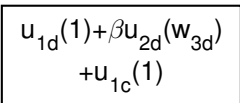

$u_{2 d}\left(w_{3 d}\right)+u_{2 c}\left(w_{3 c}\right)$

Figure 4. Decode-compress-forward transmission for the AWGN full-duplex relay channel over four blocks.

Each of the codewords $U_{1 d}, U_{2 d}, U_{1 c}$ and $U_{2 c}$ are normally distributed. The term $\beta U_{2 d}$ represents the assistance that the source provides for the relay destination transmission. This assistance depends on the correlation between $U_{1 d}+\beta U_{2 d}$ and $U_{2 d}$, which is denoted by $\rho$.

Remark 1. In DCF, normally one can optimize the power allocation of each code at the source and the relay. We fix the power of one of the DF signals $U_{1 d}$ and the $C F$ signal $U_{1 c}$. The power of the remaining signals can be obtained as a function of the power of $U_{1 d}$ and $U_{1 c}$ and the power constraint at the source and relay nodes. Consequently, the design variables of the rate maximization problem become the power of $U_{1 d}, U_{1 c}$ and the correlation $\rho$.

Assuming that the power of $U_{1 d}$ is $P_{1 d}$ and the power of $U_{1 c}$ is $P_{1 c}$, the power of $U_{2 d}$ is then given by

$$
P_{2 d}=\frac{P_{1}-P_{1 d}-P_{1 c}}{\beta^{2}}
$$

and, consequently, the power of $U_{2 c}$ is

$$
\begin{aligned}
P_{2 c} & =P_{2}-P_{2 d} \\
& =P_{2}-\frac{P_{1}-P_{1 d}-P_{1 c}}{\beta^{2}},
\end{aligned}
$$

where

$$
\begin{gathered}
\rho=\frac{E\left[\left(U_{1 d}+\beta U_{2 d}\right) U_{2 d}\right]}{\sqrt{\left(P_{1 d}+\beta^{2} P_{2 d}\right) P_{2 d}}}, \\
\beta=\sqrt{\frac{\rho^{2} P_{1 d} P_{2 d}}{P_{2 d}^{2}\left(1-\rho^{2}\right)}} .
\end{gathered}
$$

The signals $Y_{2}, \hat{Y}_{2}$ and $Y_{3}$ are given by

$$
\begin{aligned}
& Y_{2}=H_{12} X_{1}+n_{2}, \\
& \hat{Y}_{2}=Y_{2}+\hat{n}, \\
& Y_{3}=H_{13} X_{1}+H_{23} X_{2}+n_{3},
\end{aligned}
$$

where $n_{2}, n_{3}$ and $\hat{n}$ are zero mean Gaussian noise with variance $\sigma_{2}^{2}, \sigma_{3}^{2}$ and $\hat{N}$, respectively. 
Based on the previous characterization for each of the distributions of the variables involved in calculating the transmission rate, the achievable rate for the AWGN DCF relay is as follows:

$$
\begin{aligned}
R \leq \min \{ & \frac{1}{2} \log \left(1+\frac{\left|H_{12}\right|^{2} P_{1 d}}{\left|H_{12}\right|^{2} P_{1 c}+\sigma_{2}^{2}}\right), \\
& \left.\frac{1}{2} \log \left(1+\frac{\left(P_{1 d}+\beta^{2} P_{2 c}\right)\left|H_{13}\right|^{2}}{\sigma_{3}^{2}}+\frac{P_{2 d}\left|H_{23}\right|^{2}}{\sigma_{3}^{2}}+2 \rho \sqrt{\frac{\left(P_{1 d}+\beta^{2} P_{2}\right) P_{2 d}\left|H_{13}\right|^{2}\left|H_{23}\right|^{2}}{\sigma_{3}^{4}}}\right)\right\} \\
& +\frac{1}{2} \log \left(\frac{\left(\left|H_{12}\right|^{2} P_{1 c}+\sigma_{3}^{2}+\hat{N}\right)\left(\left|H_{13}\right|^{2} P_{1 c}+\sigma_{3}^{2}\right)-\left(\left|H_{12}\right|^{2}\left|H_{13}\right|^{2}\right) P_{1 c}^{2}}{\left(\sigma_{3}^{2}+\hat{N}\right) \sigma_{3}^{2}}\right),
\end{aligned}
$$

where

$$
\hat{N}=\frac{\left(\left|H_{12}\right|^{2} P_{1 c}+\sigma_{2}^{2}\right)\left(\left|H_{13}\right|^{2}\left(P_{1 d}+P_{1 c}\right)+\sigma_{2}^{2}\right)}{\left|H_{23}\right|^{2} P_{2 c}} .
$$

To illustrate the performance of DCF as a function of the location of the relay, we adopt the illustration used in [3] and calculate the achievable rate as a function of relay position on a line extending from the source to destination. For simplicity, a path-loss model is considered with the channel coefficient $H_{i j}=1 / d_{i j}^{\alpha}$, where $d_{i j}$ is the distance between node $i$ and node $j$ and $\alpha$ is the path-loss coefficient, which is usually between 2 and 4 . The distance between the source and the destination is fixed to $d_{13}=1$ while the distances $d_{12}$ and $d_{23}$ depend on the relay location where $d_{23}=1-d_{12}$. In Figure 5 , the achievable rate of different transmission techniques are shown as a function of the distance between the source and the relay $d_{12}$.

Figure 5 shows that the achievable rate of DCF in the AWGN relay channel reduces to either DF rate or CF rate. In other words, optimizing DCF results in either DF or CF.

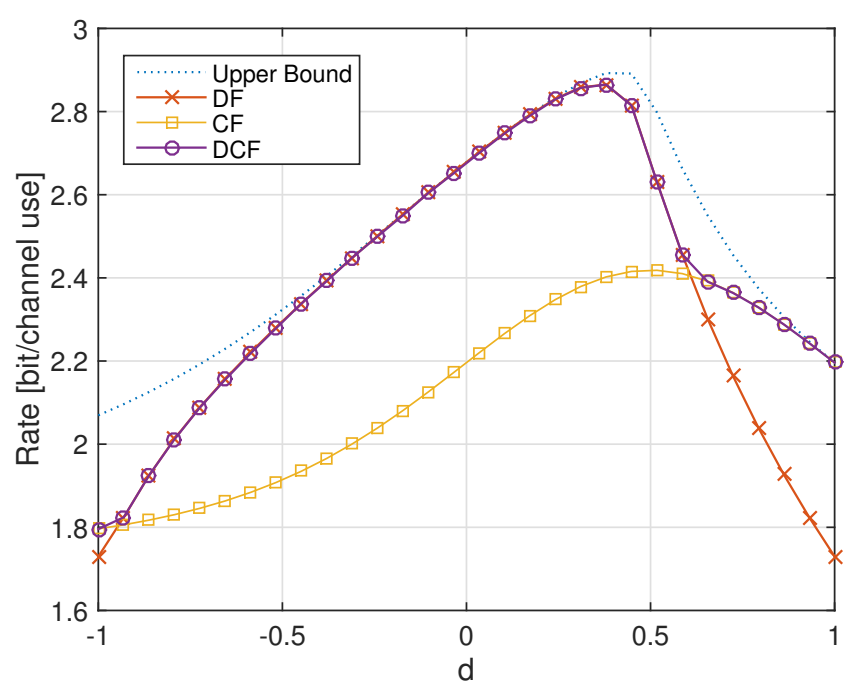

Figure 5. The achievable rate of different transmission techniques in the AWGN full-duplex relay channel.

\subsection{Constellation-Constrained Full-Duplex Relay}

For the discrete input AWGN relay channel, the rate expressions in Theorem 1 can be calculated via numerical integrations. The optimizing distribution may require an exhaustive search. As shown in many point-to-point and multi-user scenarios [33,34], when the constellation becomes large enough, the achievable rate under a constrained constellation becomes very close to the Gaussian input rate. 
However, the main difficulty comes from the restriction in Equation (21), which is now even harder to satisfy since the mutual information $I\left(X_{2} ; Y_{3}\right)$ is no longer equal to

$$
\frac{1}{2} \log \left(1+\frac{\left|H_{23}\right|^{2} P_{2}}{\sigma_{3}}\right)
$$

and is limited by the cardinality of the input size $\left|X_{2}\right|$. The exact value of the constellation constrained capacity [35], $I\left(X_{2} ; Y_{3}\right)$, can be obtained from

$$
I\left(X_{2} ; Y_{3}\right)=\max _{P_{X_{2}}\left(x_{2}\right)} \sum_{X_{2}} P_{X_{2}}\left(x_{2}\right) \int_{y_{3}} P_{Y_{3} \mid X_{2}}\left(y_{3} \mid x_{2}\right) \log \left(\frac{P_{Y_{3} \mid X_{2}}\left(y_{3} \mid x_{2}\right)}{P_{Y_{3}}\left(y_{3}\right)}\right) d y_{3} .
$$

An accurate approximation for $I\left(X_{2} ; Y_{3}\right)$ under constrained constellation can be obtained using the Blahut-Arimoto algorithm [36,37]. Constellation constrained point-to-point channel capacity for various constellations is shown in Figure 6.

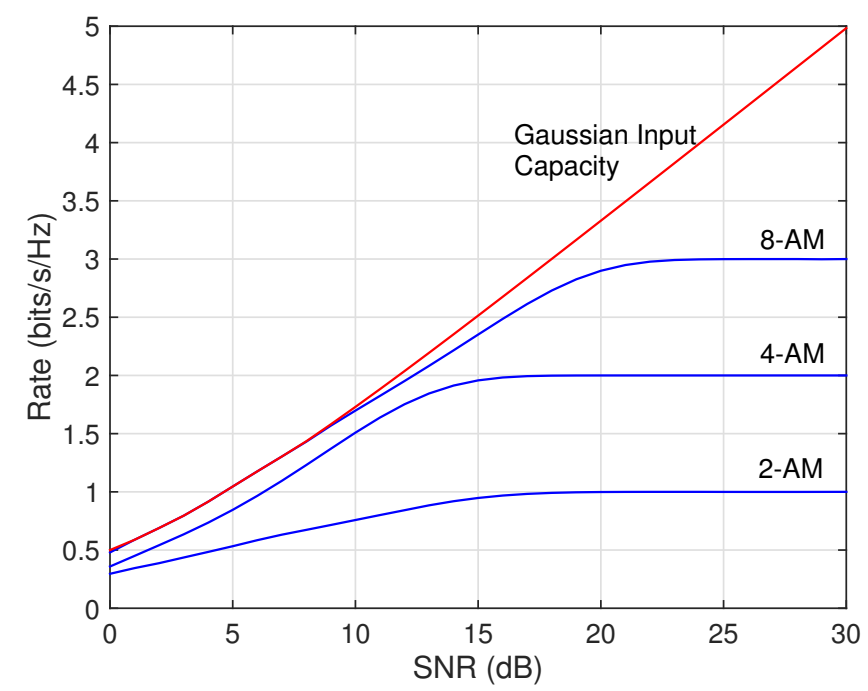

Figure 6. The constellation constrained capacity of several constellations in the point-to-point AWGN channel compared with the Gaussian input capacity.

By calculating the value of $I\left(X_{2} ; Y_{3}\right)$, one can find the achievable rate of compress-forward and DCF. In the following, an upper bound is provided on the achievable rate of DCF under discrete relay-destination input $X_{2}$. The upper bound is based on bounding $I\left(X_{2} ; Y_{3}\right)$ by either the cardinality of $X_{2}$ or the Gaussian capacity

$$
I\left(X_{2} ; Y_{3}\right) \leq \min \left\{\left|X_{2}\right|, \frac{1}{2} \log \left(1+\frac{\left|H_{23}\right|^{2} P_{2}}{\sigma_{3}^{2}}\right)\right\} .
$$

Therefore, the constraint in Equation (21) becomes

$$
I\left(Y_{2} ; \hat{Y}_{2} \mid X_{2}, U_{1 d}\right) \leq \min \left\{\left|X_{2}\right|, \frac{1}{2} \log \left(1+\frac{\left|H_{23}\right|^{2} P_{2}}{\sigma_{3}^{2}}\right)\right\}-I\left(U_{2 d} ; Y_{3}\right) .
$$

By using the bound in Equation (36), one can find an upper bound on the DCF as follows:

$$
R \leq \min \left\{I\left(U_{1 d} ; Y_{2} \mid U_{2 d}\right), I\left(U_{1 d}, U_{2 d} ; Y_{3}\right)\right\}+I\left(X_{1} ; \hat{Y}_{2}, Y_{3} \mid X_{2}, U_{1 d}\right)
$$


subject to

$$
I\left(Y_{2} ; \hat{Y}_{2} \mid X_{2}, U_{1 d}\right) \leq \min \left\{\frac{1}{2} \log \left(1+\frac{\left|H_{23}\right|^{2} P_{2}}{\sigma_{3}^{2}}\right),\left|X_{2}\right|\right\}-I\left(U_{2 d} ; Y_{3}\right)
$$

The capacity of the decode-compress-forward technique can be obtained by evaluating these expressions using numerical integrations and an exhaustive search for the optimal distribution.

In a similar manner to Section 3.2, the achievable rate for different strategies is shown in Figure 7, showing that when the relay is close to the destination, CF suffers a rate penalty while DCF does not.

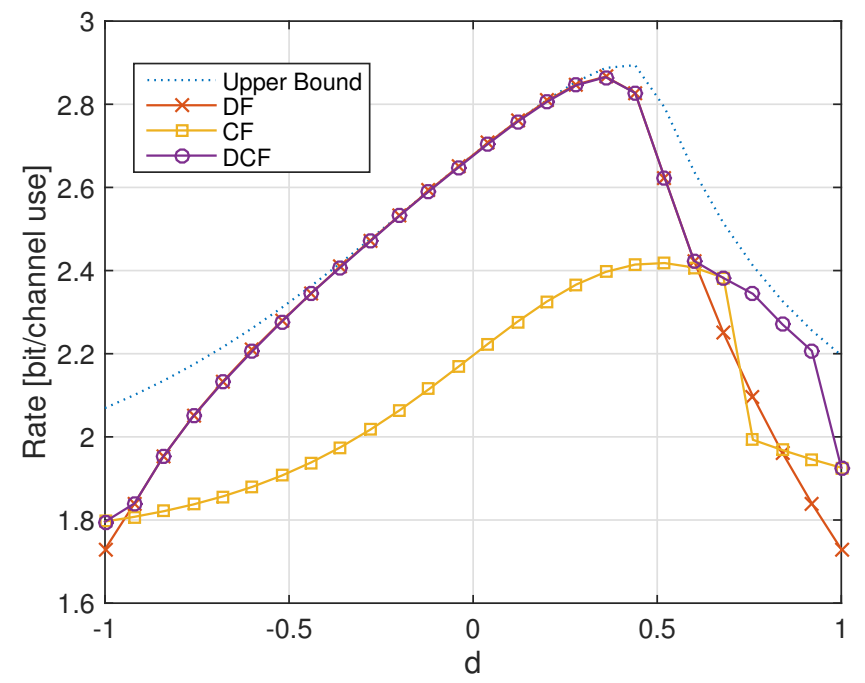

Figure 7. The achievable rate of different transmission techniques in the AWGN relay channel under a constrained constellation of 16-PAM at the source and 4-PAM at the relay.

Remark 2. Compress-forward works well when the source-relay channel is weak, but the relay destination channel is strong so that an estimate of $Y_{2}$ can be communicated with the destination. If the relay-destination channel supports insufficient rate (either because of channel gain or limitation of constellation) communicating an estimate of $Y_{2}$ is not an attractive strategy for the relay. This can in part explain the effect of the constellation constraint on the performance of compress-forward.

Clearly, the relay channel model in Figure 7 does not represent every possible scenario. We show two more results in Table 1 that cannot be observed in Figure 7.

Table 1. The achievable rate of different strategies in the relay channel under different values of channel coefficients.

\begin{tabular}{cccccccc}
\hline $\boldsymbol{H}_{\mathbf{1 2}}$ & $\boldsymbol{H}_{\mathbf{2 3}}$ & $\boldsymbol{H}_{\mathbf{1 3}}$ & $\boldsymbol{R}_{\text {No Relay }}$ & $\boldsymbol{R}_{\boldsymbol{D F}}$ & $\boldsymbol{R}_{\boldsymbol{C F}}$ & $\boldsymbol{R}_{\boldsymbol{D C F}}$ & Upper Bound \\
\hline 1 & 100 & 1 & 1.7 & 1.7 & 1.9 & 2.1 & 2.19 \\
1 & 100 & 2 & 2.19 & 1.7 & 2.3 & 2.45 & 2.47 \\
\hline
\end{tabular}

\section{Multilevel Decode-Compress-Forward}

The proposed multilevel coding is shown in Figure 8, over one transmission block. The message at the source, $W^{(t)}$, is divided into two parts, $W_{d}^{(t)}$ which is to be transmitted using decode-forward and $W_{c}^{(t)}$ which is to be transmitted using compress-forward. As shown in the figures, each level at the source and the relay is responsible for either DF or CF. 

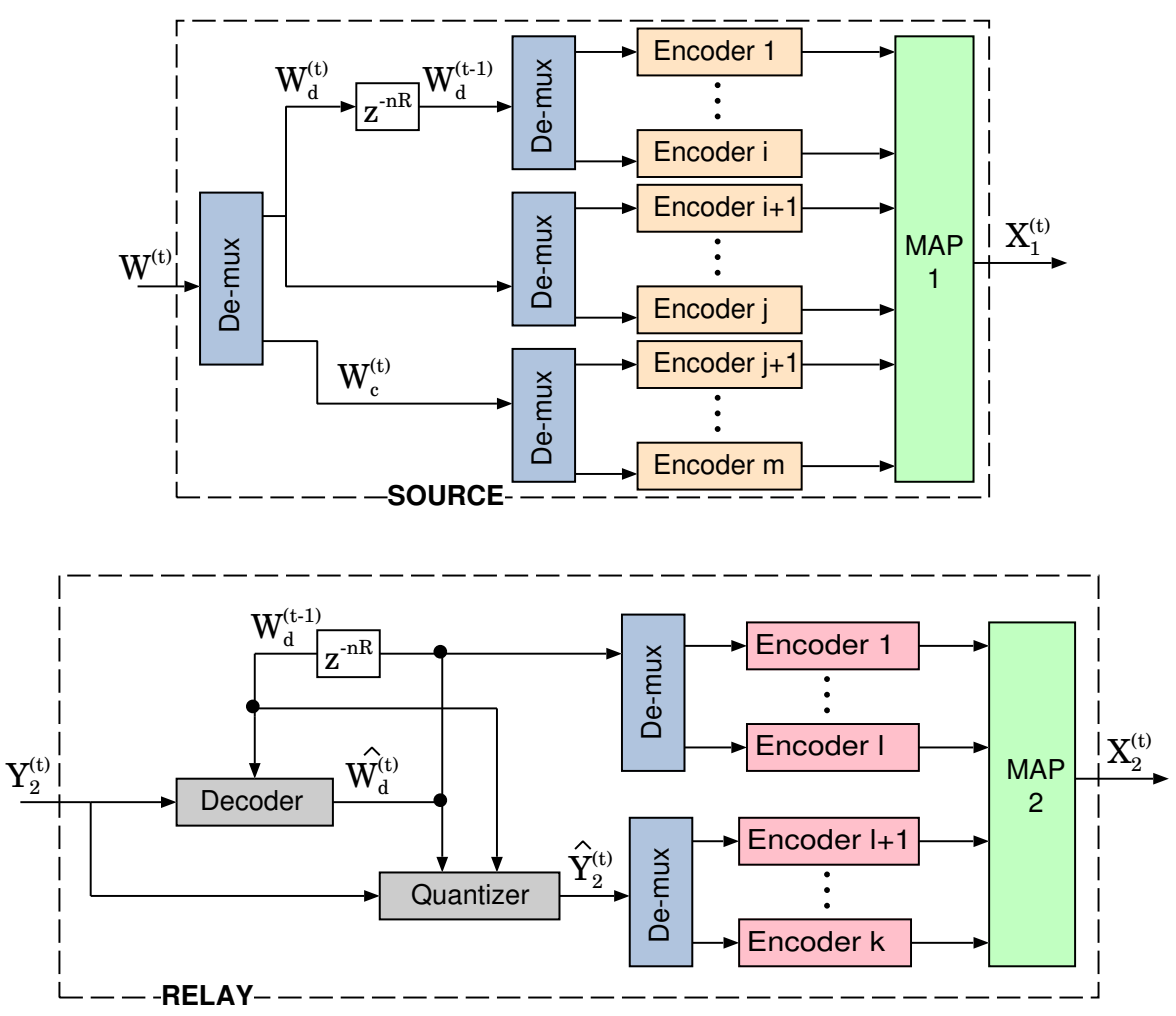

Figure 8. Multilevel coding for full-duplex decode-compress-forward relay.

\section{Relay transmission:}

The levels at the relay are divided into two sets. The first set sends the message $W_{d}^{(t-1)}$. The second set sends the quantized version of $Y_{2}$ after removing the effect of $W_{d}$, namely, $\hat{Y}_{2}$.

\section{Source transmission:}

The levels at the source are divided into three sets. The first set sends the message $W_{d}^{(t-1)}$ cooperatively with the relay to the destination node. The second set sends the message $W_{d}^{(t)}$ to be decoded at the relay. The third set of levels sends the message $W_{c}^{(t)}$.

The achievable rate of the multilevel coding DCF is given by

$$
\begin{aligned}
\left.R \leq \max _{\hat{B}, \bar{B}, \hat{C}, \bar{C}} \min \left\{I\left(\hat{B} ; Y_{2} \mid \hat{C}\right)\right\}, I\left(\hat{B}, \hat{C} ; Y_{3}\right)\right\} \\
+I\left(\hat{B}, \bar{B} ; \hat{Y}_{2}, Y_{3} \mid \hat{C}, \bar{C}, \hat{B}\right)
\end{aligned}
$$

subject to

$$
I\left(Y_{2} ; \hat{Y}_{2} \mid \hat{C}, \bar{C}\right) \leq I\left(\hat{C}, \bar{C} ; Y_{3}\right)-I\left(\hat{C} ; Y_{3}\right)
$$

where $\hat{B}=\left[B_{1}, \ldots, B_{j}\right]$ are the source levels that are responsible for the decode-forward part. $\bar{B}=\left[B_{j+1} \ldots B_{m}\right]$ are the source levels responsible for the compress-forward part. Similarly, $\hat{C}=\left[C_{1}, \ldots C_{l}\right]$ and $\bar{C}=\left[C_{l+1}, \ldots C_{m}\right]$ are the relay levels responsible for the DF part and $C F$ part, respectively.

The question now is how to allocate the rate in each level at the source and the relay. This is determined through the following steps.

1. The optimal function of each level (being a DF or a CF level) at the source and the relay depends on the constellation and the channel conditions. Using an exhaustive search to opti ize (39), 
one can find the best set of levels for decode-forward and for compress-forward at the source and the relay.

2. The number of bits of $W_{d}^{(t)}$ and $W_{c}^{(t)}$ depends on the rate of the decode-forward and compress-forward components given by

$$
\left.R_{d}=\min \left\{I\left(\hat{B} ; Y_{2} \mid \hat{C}\right)\right\}+I\left(\hat{B}, \hat{C} ; Y_{3}\right)\right\}
$$

and

$$
R_{c}=I\left(\hat{B}, \bar{B} ; \hat{Y}_{2}, Y_{3} \mid \hat{C}, \bar{C}, \hat{B}\right),
$$

respectively.

Thus, $W_{d}$ has rate $n R_{d}$ and $W_{c}$ has rate $n R_{c}$.

Remark 3. In the proposed multilevel DCF, we assume that each level is limited to either DF or CF. Extensive simulations show that this restriction has a negligible effect on achievable rates.

Remark 4. In addition to level-assignment, other labeling variations are also possible, e.g., natural versus Gray labeling. Simulations in the sequel indicate that natural labeling is in general preferable to Gray labeling.

\section{Simulations}

For the decode-forward components, we use the DVB-S2 LDPC codes. For the compress-forward components, we use the polar codes designed for compress-forward by Blasco-Serrano [24,25]. The optimal code rate of each level is obtained via an exhaustive search to maximize the rate. The block length of the component codes is $n=64 k$. Both the relay and destination nodes used belief propagation decoding at each level where the maximum number of iterations is set to 50 .

For simplicity of notation, in the following, we use $P(x)$ to denote $P_{X}(x)$. While decoding level $i$ of the signal $X_{1}$ at the relay, the relay knows two parts of $X_{1}$ already. The first is the source assistance to the relay and the second is the output of the preceding decoders at the relay, assuming correct decoding. Therefore, the log-likelihood ratio (LLR) of level $i$ at the relay is

$$
L L R_{r}=\log \frac{P\left(y_{2} \mid c^{k}, b^{i-1}, 0\right)}{P\left(y_{2} \mid c^{k}, b^{i-1}, 1\right)}
$$

where

$$
P\left(y_{2} \mid \hat{c}, b^{i-1}, b_{i}\right)=\frac{1}{P\left(\hat{c}, b^{i-1}, b_{i}\right)} \sum_{b_{i+1}^{m}} P\left(y_{2} \mid \hat{c}, \hat{b}\right)
$$

The relay removes the effect of $W_{d}^{(t-1)}$ and $W_{d}^{(t)}$ from $Y_{2}$ by first encoding them and removing their effect from $X_{1}$.

The decoding at the destination node is performed as follows: after the last transmission block, the destination decodes the decode-forward signal from the relay and then decode the signal from the source node. The LLR of level $i$ of the relay at the destination node is

$$
L L R_{R D}=\log \frac{P\left(y_{3} \mid c^{i-1}, 0\right)}{P\left(y_{3} \mid c^{i-1}, 1\right)},
$$

where

$$
P\left(y_{3} \mid c^{i-1}, c_{i}\right)=\frac{1}{P\left(c^{i-1}, c_{i}\right)} \sum_{b^{m}, c_{i+1}^{m}} P\left(y_{3} \mid b^{m}, c^{m}\right) .
$$


The next step is to decode the signal from the source given the transmitted signal from the relay with

$$
L L R_{S D}=\log \frac{P\left(y_{3} \mid c^{m}, b^{i-1}, 0\right)}{P\left(y_{3} \mid c^{m}, b^{i-1}, 1\right)}
$$

where

$$
P\left(y_{3} \mid c^{m}, b^{i-1}, b_{i}\right)=\frac{1}{P\left(c^{m}, b^{i-1}, b_{i}\right)} \sum_{b_{i+1}^{m}} P\left(y_{3} \mid b^{m}, c^{m}\right) .
$$

In each of the error plots, a capacity threshold is marked that corresponds to the relay DCF constellation constrained capacity. The source and relay powers are identical throughout all simulations, enabling the use of a single scale for power $(\mathrm{dB})$ in the error curves.

Figure 9 shows the bit error probability and frame error probability for a 16-QAM source and QPSK relay. The figure compares the performance of DCF under different labelings. The system model is the same as the one considered in Figure 7 where the source, relay and destination are all on one line. The distance between the source and relay is 0.8 , the distance between the relay and destination is 0.2 and the distance between the source and destination is 1 . The path-loss coefficient is $\alpha=2$.

The total transmission rate is $2.2 \mathrm{bits} / \mathrm{s} / \mathrm{Hz}$. The four levels of the source operate as follows: the most significant bit transmits the same information of the most significant bit of the relay to provide a beamforming gain to the relay-destination transmission. The following two levels at the source transmit new information to be decodable at the relay while the last level transmits a compress-forward component.

Figure 10 shows the bit error probability and frame error probability for a 16-QAM source and QPSK relay. The model that is considered this time is given by the channel gains, more specifically, $H_{12}=1, H_{23}=100$ and $H_{13}=1$. This figure compares the proposed DCF with DF and CF. The rate is 2 bits/s/Hz. The figure shows that DCF outperforms DF and CF.

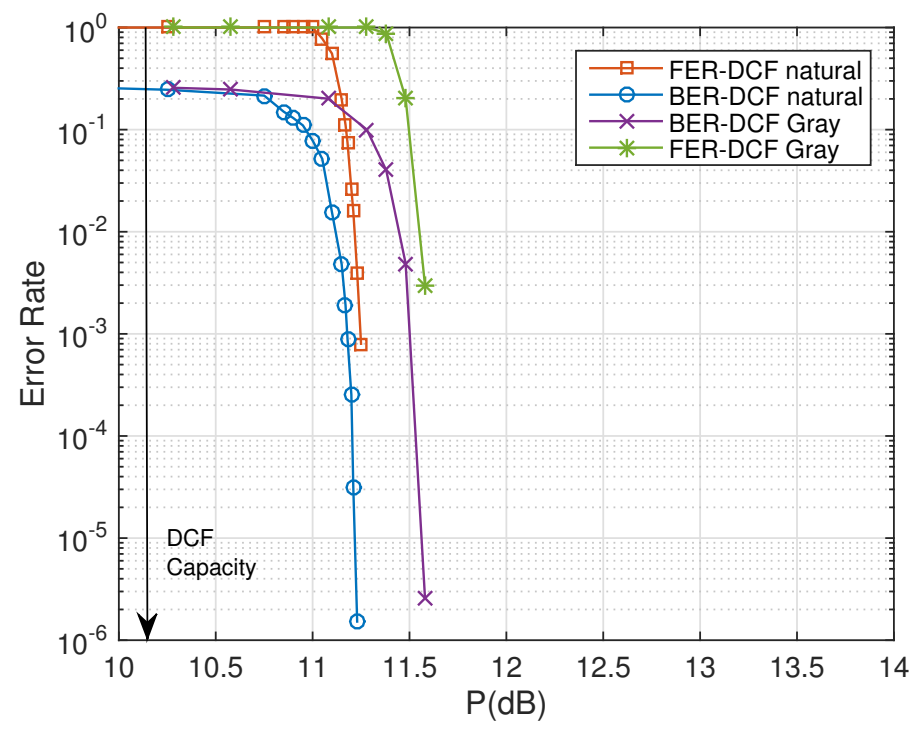

Figure 9. Performance of multilevel superposition for 8-PAM constellation when the relay is located at $d=2.5$. 


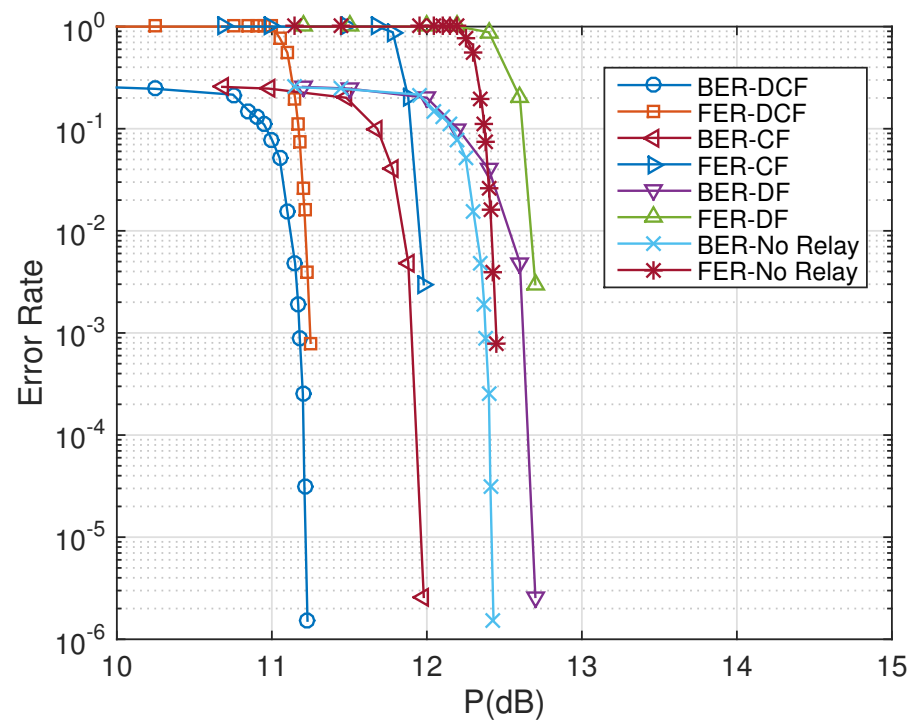

Figure 10. Performance of multilevel DCF vs. DF, CF, and no-relay.

\section{Conclusions}

This work studies the decode-compress-forward protocol, highlighting scenarios where it exceeds the rates available via decode-forward and compress-forward in the constellation constrained full-duplex relay channel. Furthermore, this work presents a multilevel coding strategy for the decode-compress-forward protocol. The proposed multilevel coding was shown to approach the rates achieved by decode-compress-forward in the constellation constrained AWGN full-duplex relay channel.

Acknowledgments: This work was supported in part by the grant CIF1219065 and ECCS1546969 from the National Science Foundation.

Author Contributions: Aria Nosratinia conceived the multilevel approach to multi-node coded modulation and contributed to mathematical formulation and proofs. Ahmed Attia Abotabl contributed to mathematical formulation, proofs, and simulations.

Conflicts of Interest: The authors declare no conflict of interest.

\section{References}

1. Van Der Meulen, E.C. Three-terminal communication channels. Adv. Appl. Probab. 1971, 3, 120-154.

2. Cover, T.; Gamal, A. Capacity theorems for the relay channel. IEEE Trans. Inf. Theory 1979, 25, 572-584.

3. Kramer, G.; Marić, I.; Yates, R.D. Cooperative communications. Found. Trends Netw. 2007, 1, $271-425$.

4. Kramer, G.; Gastpar, M.; Gupta, P. Cooperative Strategies and Capacity Theorems for Relay Networks. IEEE Trans. Inf. Theory 2005, 51, 3037-3063.

5. Laneman, J.N. Cooperative Diversity in Wireless Networks: Algorithms and Architectures. Ph.D. Thesis, Massachusetts Institute of Technology, Cambridge, MA, USA, 2002.

6. Lee, S.H.; Chung, S.Y. When is compress-and-forward optimal? In Proceedings of the Information Theory and Applications Workshop (ITA), San Diego, CA, USA, 31 January-5 February 2010; pp. 1-3.

7. Bao, X.; Li, J. Efficient Message Relaying for Wireless User Cooperation: Decode-Amplify-Forward (DAF) and Hybrid DAF and Coded-Cooperation. IEEE Trans. Wirel. Commun. 2007, 6, 3975-3984.

8. Haghighat, J.; Hamouda, W. Decode-Compress-and-Forward with Selective-Cooperation for Relay Networks. IEEE Commun. Lett. 2012, 16, 378-381.

9. Chong, H.F.; Motani, M.; Garg, H.K. Generalized Backward Decoding Strategies for the Relay Channel. IEEE Trans. Inf. Theory 2007, 53, 394-401. 
10. Imai, H.; Hirakawa, S. A new multilevel coding method using error-correcting codes. IEEE Trans. Inf. Theory 1977, 23, 371-377.

11. Wachsmann, U.; Fischer, R.F.H.; Huber, J. Multilevel codes: Theoretical concepts and practical design rules. IEEE Trans. Inf. Theory 1999, 45, 1361-1391.

12. Huber, J.; Wachsmann, U. Capacities of equivalent channels in multilevel coding schemes. Electron. Lett. 1994, 30, 557-558.

13. Huber, J.; Wachsmann, U. Design of multilevel codes. In Proceedings of the IEEE Information Theory Workshop (ITW), Rydzyna, Poland, 25-29 June 1995.

14. Duan, L.; Rimoldi, B.; Urbanke, R. Approaching the AWGN channel capacity without active shaping. In Proceedings of the IEEE International Symposium on Information Theory ISIT, Ulm, Germany, 29 June-4 July 1997; p. 374.

15. Lampe, L.H.J.; Fischer, R.F.H.; Schober, R. Multilevel coding for multiple-antenna transmission. IEEE Int. Symp. Inf. Theory 2002, 3, 203-208.

16. Yeung, R. Multilevel diversity coding with distortion. IEEE Trans. Inf. Theory 1995, 41, 412-422.

17. Roche, J.; Yeung, R.; Hau, K.P. Symmetrical multilevel diversity coding. IEEE Trans. Inf. Theory 1997, 43, 1059-1064.

18. Mohajer, S.; Tian, C.; Diggavi, S. Asymmetric Multilevel Diversity Coding and Asymmetric Gaussian Multiple Descriptions. IEEE Trans. Inf. Theory 2010, 56, 4367-4387.

19. Jiang, J.; Marukala, N.; Liu, T. Symmetrical Multilevel Diversity Coding and Subset Entropy Inequalities. IEEE Trans. Inf. Theory 2014, 60, 84-103.

20. Abdel-Ghaffar, K.; Hassner, M. Multilevel error-control codes for data storage channels. IEEE Trans. Inf. Theory 1991, 37, 735-741.

21. Hern, B.; Narayanan, K. Multilevel Coding Schemes for Compute-and-Forward With Flexible Decoding. IEEE Trans. Inf. Theory 2013, 59, 7613-7631.

22. Chen, Z.; Xia, B.; Hu, Z.; Liu, H. Design and Analysis of Multi-Level Physical-Layer Network Coding for Gaussian Two-Way Relay Channels. IEEE Trans. Commun. 2014, 62, 1803-1817.

23. Morello, A.; Mignone, V. DVB-S2: The second generation standard for satellite broad-band services. Proc. IEEE 2006, 94, 210-227.

24. Blasco-Serrano, R. Coding Strategies for Compress-and-Forward Relaying. Ph.D. Thesis, Kungliga Tekniska Högskolan (KTH), Stockholm, Sweden, 2010.

25. Blasco-Serrano, R.; Thobaben, R.; Rathi, V.; Skoglund, M. Polar codes for compress-and-forward in binary relay channels. In Proceedings of the Forty Fourth Asilomar Conference on Signals, Systems and Computers, Pacific Grove, CA, USA, 7-10 November 2010; pp. 1743-1747.

26. Gamal, A.E.; Kim, Y. Network Information Theory; Cambridge University Press: Cambridge, UK, 2012.

27. Duarte, M. Full-Duplex Wireless: Design, Implementation and Characterization. Ph.D. Thesis, Rice University, Houston, TX, USA, 2012.

28. Shende, N.; Gurbuz, O.; Erkip, E. Half-duplex or full-duplex relaying: A capacity analysis under self-interference. In Proceedings of the 47th Annual Conference on Information Sciences and Systems (CISS), Baltimore, MD, USA, 20-22 March 2013; pp. 1-6.

29. Ahmed, E.; Eltawil, A.M. All-Digital Self-Interference Cancellation Technique for Full-Duplex Systems. IEEE Trans. Wirel. Commun. 2015, 14, 3519-3532.

30. Alexandris, K.; Balatsoukas-Stimming, A.; Burg, A. Measurement-based characterization of residual self-interference on a full-duplex MIMO testbed. In Proceedings of the IEEE 8th Sensor Array and Multichannel Signal Processing Workshop (SAM), A Coruna, Spain, 22-25 June 2014; pp. 329-332.

31. Mahmood, N.H.; Ansari, I.S.; Berardinelli, G.; Mogensen, P.; Qaraqe, K.A. Analysing self interference cancellation in full duplex radios. In Proceedings of the IEEE Wireless Communications and Networking Conference, Doha, Qatar, 3-6 April 2016; pp. 1-6.

32. Ingber, A.; Feder, M. On the optimality of multilevel coding and multistage decoding. In Proceedings of the IEEE 25th Convention of Electrical and Electronics Engineers in Israel, Eilat, Israel, 3-5 December 2008; pp. 731-735.

33. Mheich, Z.; Alberge, F.; Duhamel, P. Achievable rates optimization for broadcast channels using finite size constellations under transmission constraints. EURASIP J. Wirel. Commun. Netw. 2013, 2013, 1-15. 
34. Abotabl, A.A.; Nosratinia, A. Broadcast Coded Modulation: Multilevel and Bit-Interleaved Construction. IEEE Trans. Commun. 2017, 65, 969-980.

35. Ungerboeck, G. Channel coding with multilevel/phase signals. IEEE Trans. Inf. Theory 1982, $28,55-67$.

36. Blahut, R. Computation of channel capacity and rate-distortion functions. IEEE Trans. Inf. Theory 1972, 18, 460-473.

37. Arimoto, S. An algorithm for computing the capacity of arbitrary discrete memoryless channels. IEEE Trans. Inf. Theory 1972, 18, 14-20.

(C) 2017 by the authors. Licensee MDPI, Basel, Switzerland. This article is an open access article distributed under the terms and conditions of the Creative Commons Attribution (CC BY) license (http:/ / creativecommons.org/licenses/by/4.0/). 